BULL. AUSTRAL. MATH. SOC.

VOL. $30(1984), 179-191$

\title{
TWO-SIDED LOCALIZATION IN SEMIPRIME FBN RINGS
}

\author{
M.H. UPHAM
}

\begin{abstract}
The main result of this paper is that the left- and rightquotient rings at a hereditary link closed set of prime ideals of a semiprime fully bounded Noetherian (FBN) ring coincide. This was a result already known for nonsemiprime FBN rigns, but a question left open in the semiprime case. A cornerstone of our approach is that the torsion theory determined by a link-closed hereditary set of prime ideals in an FBN ring is "nice", but not necessarily perfect. Some conditions which do produce perfect torsion theories are investigated.
\end{abstract}

\section{Introduction}

In [7], Mïller showed that for an FBN ring with locally finite links, the left and right quotient rings at a hereditary link-closed set of prime ideals coincide. Such rings include all Noetherian PI rings and all FBN rings which are not semiprime [7, Theorem 7], but the question of two-sided quotient rings in semiprime FBN rings was left open (see [7], [8]). In this paper, we show that the left and right quotient rings at a hereditary link-closed set of prime ideals of a semiprime FBN ring do coincide (Theorem 8).

Throughout $R$ denotes a fully bounded joetherian (FBN) ring on both sides. Modules are unitary right modules unless a subscript indicates a Received 16 March 1984.

Copyright Clearance Centre, Inc. Serial-fee code: 0004-9727/84 $\$ A 2.00+0.00$. 
left module. Maps are written opposite scalars. We assume familarity with the concept of a torsion theory determined by

(i) an injective module,

(ii) an incomparable (possibly infinite) set of prime ideals of $R \quad(Q \subseteq R$ is incomparable if $P, Q \in Q$ and $P \subseteq Q \Rightarrow P=Q$ )

$\tau(M)$ denotes the torsion submodule of a module $M, \Delta(M)$ denotes the quotient module of a right module $M$ and $\Lambda(M)$ denotes the quotient module of a left module $R^{M}$. If the torsion theory is not clear from the context, suitable subscripts may be added. See [4], [11] for details. When the meaning is clear from the context we shall let $\Delta$ denote $\Delta\left(R_{R}\right)$ and $\Lambda$ denote $\Lambda\left({ }_{R} R\right)$. When $R$ is FBN, there is a one-to-one correspondence between prime ideals of $R$ and indecomposable injective modules (up to isomorphism). $E_{Q}$ denotes the indecomposable injective with associated prime ideal $Q$. A set $P \subseteq$ Spec $R$ is called hereditary if $P \subseteq Q \in P \Rightarrow P \in P$. The torsion theories over an RBN ring are in one-toone correspondence with the hereditary sets of prime ideals, these being the closed prime ideals in the torsion theory. Every dense right ideal contains a product of dense prime ideals [1].

We also assume familiarity with the ideas of Krull dimension, critical module, basic series, and so on, developed in [3] as well as the definitions of a short right link $P \sim Q$ and a long right link $P \sim>\sim Q$ between prime ideals $P$ and $Q$. (See [6].) Call $P \subseteq$ Spec $R$ link-closed if $P \sim Q$ and one of $P, Q$ in $P$ implies the other also belongs to $P$.

We shall begin with a general torsion-theoretic result and then consider two-sided localization in certain factor rings. Out of this comes a useful connection between $\Lambda(R)$ and $\Delta(R)$ which will then be applied to the question of two-sided localization over a semiprime FBN ring $R$. Finally we offer some contributions to the study of perfect torsion theories and link-closed hereditary subsets of $\operatorname{Spec}(R)$. It is known that a link-closed hereditary subset of $\operatorname{Spec}(R)$ does not necessarily produce a perfect torsion theory. We present some sufficient conditions for this to happen.

We shall require the following result concerning a torsion theory over 
an RBN ring $R$. If $\tau$ is a torsion radical on Mod-R and $A$ is a $\tau$-closed right ideal of $R$, then $\tau(R) \subseteq A$. Hence $A / \tau(A)=A / \tau(R) \subseteq R / \tau(R)$ and $\Delta(A) \subseteq \Delta(R)$. Furthermore $\Delta(A) \cap(R / \tau(R))=A / \tau(R)$.

PROPOSITION 1 . Let 2 be an incomparable link closed subset of Spec $R$ where $R$ is FBN. Then

(a) every 2-torsion-free factor module of $\Delta_{2}(R)$ is divisible,

(b) $\Delta_{Q^{(R) / \Delta}} Q^{(Q)} \approx \Delta_{2}(R / Q)$ for alz $Q \in Q$,

(c) $\Delta_{2}(Q)$ is a prime ideal of $\Delta_{2}(R)$ for all $Q \in Q$.

Proof. Let $\Delta$ denote $\Delta_{Q}(R)$.

(a) We first show that any basic submodule of a finitely generated 2-torsion-free module $X$ is closed in the divisible hull of $X, D(X)$. Let $C$ be a basic submodule of some finitely generated, 2-torsion-free module $X$ (that is, $C$ is maximal among the $\alpha$-critical submodules of $X$ where $\alpha$ is the least possible Krull dimension of a nonzero submodule of $X)$. Suppose $t \in D(X) \backslash C$ and $t D \subseteq C$ where $D$ is a dense ideal. $C$ is uniform [3] and $X$ is torsion-free. Hence $\mathrm{Ann}_{R} C$ is a prime ideal $P \subseteq Q$ for some $Q \in 2$. If $t R \in t R \backslash C, \operatorname{tr} D=0$ would imply $t r=0$, contradiction. Hence $t r+C$ is an essential, hence uniform extension of $C$. If $(t R+C) / C$ is not critical, it contains a basic submodule $C_{1} / C$ [3]. Let $P^{\prime}=\operatorname{Ann}_{R}\left(C_{1} / C\right)$. Since $P^{\prime} \supseteq D, P^{\prime}$ is 2-dense. Now $0 \varsubsetneqq C \varsubsetneqq C_{1}$ is a basic series for $C_{1}$. So

$$
K-\operatorname{dim}(R / P)=K-\operatorname{dim}(C) \leq K-\operatorname{dim}\left(R / P^{\prime}\right)
$$

[3, Theorem 3.4]. On the other hand, the canonical epimorphism $C_{1} \rightarrow C_{1} / C \rightarrow 0$ induces a nonzero homomorphism $E_{P} \rightarrow E_{P^{\prime}}$. This implies $P^{\prime}$ contains some prime ideal $B$ with $P \leadsto \ldots \sim B$ [6, Lemma 3]. Hence $K-\operatorname{dim}\left(R / P^{\prime}\right) \leq K-\operatorname{dim}(R / B)=K-\operatorname{dim}(R / P)$. In other words, we have a link between closed prime ideal $P$ and dense prime ideal $P^{\prime}$ which contradicts [7, Corollary 3].

Now consider the module $X=\Delta / Y$ where $y_{R}$ is a 2-closed submodule of $\Delta_{R}$. Then $Y_{R}$ is torsion-free divisible. Thus $Y \Delta=Y$ and we may 
assume $X=x \Delta$. We proceed by induction on the Krull-composition length of $x R$.

If $x R$ is already basic, we have shown $x R$ is closed in $D(x R)$, hence is divisible. Then $x \Delta \subseteq D(x R)=x R$. If $x R$ has Krull-composition series of length $n$, say $0=B_{0} \varsubsetneqq B_{1} \varsubsetneqq B_{2} \varsubsetneqq \cdots \varsubsetneqq B_{n}=x k$ then, by induction assumption, $x \Delta / B_{\perp}$ is divisible. Also $B_{1}$ is divisible. Since divisible modules are closed under group extensions, $x \Delta$ is divisible.

(b) We first show that $\Delta(R) / \Delta(Q)$ is a torsion-free $k$-module. If for some $x \in \Delta(R)$ there exists a dense (right) ideal $D$ such that $x D \subseteq \Delta(Q)$, since $D$ is finitely generated, we can find a dense ideal $D^{\prime}$ such that $x D D^{\prime} \subseteq Q / \tau(R)$. This implies $x \in \Delta(Q)$. By $(a), \Delta(R) / \Delta(Q)$ is torsion-free divisible. The canonical homomorphism $m: R / Q \rightarrow \Delta(R) / \Delta(Q)$ is an essential monomorphism whose image is dense in $\Delta(R) / \Delta(Q)$. Hence $\Delta(R / Q)=\Delta(R) / \Delta(Q)$.

(c) Suppose $x \Delta(R) y \subseteq \Delta(Q)$ for elements $x, y \in \Delta(R)$. Let $D_{x}, D_{y}$ be dense ideals such that $x D_{x}$ and $y D_{y} \subseteq R / \tau(R)$. I'hen $x D_{x} y D_{y} \subseteq Q / \tau(R)$. Either $\quad x D_{x} \subseteq Q / \Delta(R)$ and $x \in \Delta(Q)$ or $y D_{y} \subseteq Q / \tau(R)$ and $y \in \Delta(Q)$.

REMARK. Lambek [4] calls an injective module $I$ "nice" if the localization at $I$ satisfies property (a) of Proposition 1 . It is clear that if the torsion theory determined by $I$ is perfect, then $I$ is certainly nice. The converse is not generally true. Indeed, if a ring $R$ is right hereditary, every injective module is nice, [4, Example 1, p. 68]. However, if $R$ is hereditary but non-Noetherian, then any torsion theory whose filter of dense right ideals contains a non-finitely generated right ideal yields a nice injective whose corresponding torsion theory is not perfect.

\section{Two-sided localization in factor rings}

In this section we assume that $R$ is FBN, not necessarily semiprime. We show that under suitable conditions, left- and right-quotient rings coincide for certain factor rings of $R$.

Consider an incomparable, link-closed set $Q \subseteq \operatorname{Spec} R$. Let $\pi$ be any product of members of 2 . Then $N_{\pi}=\{P \in Q: P \supseteq \pi\}$ is finite; in 
fact, it corresponds to the set of minimal prime ideals of $R / \pi$. Since $Q$ is link-closed, $\left\{P / \pi: P \in N_{\pi}\right\}$ is link-closed [6, Theorem 5]. Hence the semiprime ideal $N_{\pi} / \pi$ (where $N_{\pi}=n\left\{P: P \in N_{\pi}\right\}$ ) is localizable and the corresponding torsion theory is induced by the localizable multiplicative set of regular elements modulo $N_{\pi} / \pi, C_{R / \pi}\left(N_{\pi} / \pi\right)$. One consequence is that the left-and right-quotient rings of $R / \pi$ at $N_{\pi} / \pi$ coincide.

Denote this quotient ring by $Q_{\pi}$. Furthermore $N_{\pi} / \pi$ is a semiprime ideal of $R / \pi$ such that some power of $N_{\pi} / \pi$ is torsion (in fact, 0 ). Since $N_{\pi} / \pi$ is localizable, by Lambek and Michler $[5,3.3$ and 3.4$], Q_{\pi}$ is right and left Artinian and every element of $C_{R}\left(N_{\pi}\right)$ is regular modulo $\pi$. Some immediate consequences are:

LEMMA 2. Let $2, R$ and $\pi$ be as described above. Then

(a) $\tau_{2}(R) \subseteq \pi$,

(b) $\pi$ is 2-closed,

(c) $R / \pi$ is a subring of $Q_{\pi}$.

PROPOSITION 3. Suppose 2 is an incomparable link-closed subset of Spec $R \quad(R \quad F B N)$ and $\pi$ is any product of elements of 2 . Then $\Delta_{Q}(R) / \Delta_{2}(\pi) \cong Q_{\pi} \cong \Delta_{Q}(R / \pi) \quad$ (as rings)

Proof. Let $\bar{R}=R / \tau_{2}(R)$. We have $\Delta_{2}(\pi) n \bar{R}=\bar{\pi}$, hence $R / \pi \hookrightarrow \Delta_{2}(R) / \Delta_{2}(\pi)$. Now $\Delta_{2}(R) / \Delta_{2}(\pi)$ is 2 -torsion free, hence divisible by the argument of Proposition $1(b)$. Thus $\Delta_{2}(R) / \Delta_{2}(\pi) \cong \Delta_{2}(R / \pi) \subseteq Q_{\pi}$ since every 2 -dense ideal is certainly $N_{\pi^{-}}$ dense. For the reverse inclusion, given $t \in Q_{\pi}$, there exists a product of $N_{\pi} / \pi$-dense prime ideals of $R / \pi$ - say $F=\left(P_{1} P_{2} \cdots P_{k}+\pi\right) / \pi$ - such that $t F \subseteq R / \pi$. The incomparability of 2 and the definition of $N_{\pi}$ guarantee that $P_{1} \cdots P_{k}$ is 2 -dense. Hence $t \in \Delta_{2}(R / \pi) \cong \Delta_{2}(R) / \Delta_{2}(\pi)$.

COROLLARY 4. Under the same hypotheses,

$$
\Lambda_{2}(R / \pi) \cong \Lambda_{2}(R) / \Lambda_{2}(\pi) \cong Q_{\pi} \cong \Delta_{2}(R) / \Delta_{2}(\pi) \cong \Delta_{2}(R / \pi)
$$


It should be noted that the isomorphisms in Corollary 4 leave the ring $R / \pi$ fixed. Hence we obtain the following explicit rule for the isomorphism $\psi: \Delta_{Q}(R / \pi) \rightarrow Q_{\pi}$. Given $q \in \Delta_{2}(R / \pi)$, there exists a 2-dense ideal $D$ such that $q D \subseteq R / \pi$. Since $D$ is $N_{\pi}$-dense, there exists $c \in C\left(N_{\pi}\right) \cap D$ and $q c=[r]_{\pi} \in R / \pi$. Then $\psi(q c)=[r]_{\pi}$. But $\psi(q c)=\psi(q) c$. Hence $\psi(q)=[r]_{\pi}[c]_{\pi}^{-1}$.

PROPOSITION 5. Let 2 be an incomparable link-closed subset of Spec $R$ ( $R$ FBN). Let $\pi^{\prime} \subseteq \pi$ be products of members of 2 . Then there is a commutative diagram

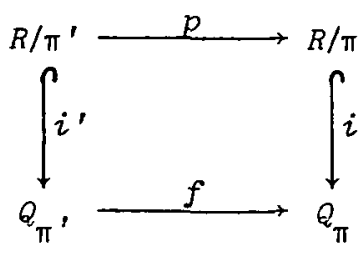

( $p$ is the canonical epimorphism and $i, i$ ' are inclusions.) Furthermore $f$ is onto.

Proof. $\|_{\pi^{\prime}}=N_{\pi} \cap A$ where $A=R$ or $A$ is an intersection of members of 2 . Consequently, $R / \pi^{\prime}$ is an $N_{\pi^{-d e n s e}}$ submodule of $Q \pi^{\prime}$. Since $Q_{\pi}$ is $N_{\pi}$-torsion free divisible, there exists a unique $f: Q_{\pi^{\prime}} \rightarrow Q$ such that $f i^{\prime}=i p$. To be explicit, given $[r]_{\pi},[c]_{\pi^{\prime}}^{-1} \in Q_{\pi}$ with $c \in \mathcal{C}\left(N_{\pi^{\prime}}\right) \subseteq \mathcal{C}\left(N_{\pi}\right)$, we must have $f\left([r]_{\pi^{\prime}}[c]_{\pi^{\prime}}^{-1}\right)=[r]_{\pi}[c]_{\pi}^{-1}$.

To verify that $f$ is onto, select any $q \in Q_{\pi}$. There exists a product $D$ of prime ideals, each non-minimal among primes containing $\pi$, such that $q D \subseteq R / \pi$. Since $Q$ is incomparable, $D$ is 2 -dense, hence $N_{\pi}$,-dense. Then there exists $c \in D \cap C\left(N_{\pi^{\prime}}\right)$ and $r \in R$ such that $q c=[r]_{\pi} \in R / \pi$. It follows that $q=[r]_{\pi}[c]_{\pi}^{-1}=f\left([r]_{\pi},[c]_{\pi^{\prime}}^{-1}\right)$.

In what follows, $E$ denotes $\left.\Theta E_{Q}: Q \in 2\right\}$ where $Q$ is an incomparable link-closed subset of Spec $R$. Since $E$ is 2-torsion free, every $R$-homomorphism $\phi: \Delta_{Q}(R) \rightarrow E$ is automatically a $\Delta_{Q}(R)-$ 
homomorphism. It follows that $E$ is $\Delta_{Q}(R)$-injective. Also, for any $Q \in Q$, since $\Delta_{Q}(R) / \Delta_{Q}(Q)$ is an essential extension of $R / Q$, it is embedded in a finite product of copies of $E$.

Consider the following topologies on $\Delta=\Delta_{2}(R)$ (cf. [5]):

(1) the E-adic, whose basic open neighborhoods of zero have the form $\operatorname{ker}(f)$ for some $f \in \operatorname{Hom}_{\Delta}\left(\Delta, E^{n}\right) \quad(n \in N)$;

(2) the $\Delta(\pi)$-adic, whose basic open neighborhoods of zero have the form $\Delta(\pi)$ where $\pi$ is some product of members of 2 .

Recall that a torsion theory is called stable if the family of torsion modules is closed under injective hulls.

PROPOSITION 6. Let $Q$ be an incomparable, link-closed set of prime $i d e a l s$ of an FBN ring $R$. Then the E-adic and the $\Delta(\pi)$-adic topologies on $\Delta=\Delta_{2}(R)$ coincide.

Proof. By Propositions 1 and 3, $\Delta(R) / \Delta(\pi)$ is right (and left) Artinian for all $\pi$ and $\Delta(R) / \Delta(Q)$ is simple Artinian for all $Q \in Q$. We claim that for any $i, \Delta\left(Q_{1} \ldots Q_{i-1}\right) \Delta\left(Q_{i}\right) \subseteq \Delta\left(Q_{1} \ldots Q_{i}\right)$. Indeed, given $x \in \Delta\left(Q_{1} \ldots Q_{i-1}\right)$ and $y \in \Delta\left(Q_{i}\right)$, let $D_{x}$ and $D_{y}$ be dense ideals such that $x D_{x} \subseteq Q_{1} \ldots Q_{i-1} / \tau(R)$ and $y D_{y} \subseteq Q_{i} / \tau(R)$. Since 2 is closed under links, the 2-torsion theory is stable [1], [11]. Hence, by [1, Theorem 1.2], there exists a dense ideal $D^{\prime}$ such that $\bar{D}_{x}\left(y D_{y}\right) \supseteq \bar{R}_{y} D_{y} \bar{D}^{\prime}$. Then $x y D_{y} D^{\prime} \subseteq x D_{x} y D_{y} \subseteq Q_{1} \ldots Q_{i} / \tau(R)$. This implies $x y \in \Delta\left(Q_{1} \ldots Q_{i}\right)$ as desired. Given a product $\pi=Q_{1} \ldots Q_{k}$ with all $Q_{i} \in \mathcal{R}$, each factor of the chain

$$
\Delta(R) \supseteq \Delta\left(Q_{1}\right) \supseteq \Delta\left(Q_{1} Q_{2}\right) \supseteq \ldots \supseteq \Delta\left(Q_{1} \ldots Q_{k}\right)
$$

is a finitely generated $\Delta(R) / \Delta\left(Q_{i}\right)$-module $(i=1,2, \ldots, k)$, hence a finite direct sum of simple $\Delta(R) / \Delta\left(Q_{i}\right)$-modules, which embeds in a finite direct product of copies of $E$. It follows that $\Delta(R) / \Delta(\pi) \subseteq E^{m}$ for some $m \in N$. 
Conversely, given $f: \Delta(R) \rightarrow E^{n}=\oplus E_{Q}^{n}$, let $f(1)=x_{1}+\ldots+x_{t}$ where each $x_{j}$ is in $E_{Q_{j}}$ for some $Q_{j} \in 2$. By considering a critical series for $x_{j} R$ [3], one sees that $x_{j} R$ is annihilated by a product of prime ideals in 2 . It follows that $f(\Delta(\pi))=f(1) \Delta(\pi)=0$ for some product $\pi$ of members of 2 .

COROLLARY 7. Under the same hypotheses, the bicommutator of $E$ is isomorphic to $S=\lim \{\Delta(R) / \Delta(\pi): \pi$ is a product of members of 2$\}$.

Proof. The conditions of [4, Propositions 2 and 3] are satisfied.

REMARK. $E$ is naturally an $S$-module under the following rule. Given $e \in E$ and $s=\left\langle\left[s_{\pi}\right]_{\Delta(\pi)}\right\rangle \in S$, where $s_{\pi} \in \Delta(R)$, choose any $\pi$ such that $e \pi=0$. Define $e s=e s \pi$. It is tedious to verify that this is a well-defined $S$-action on $E$ which extends the existing $\Delta(R)$-action. Both $\Delta(R)$ and $\Lambda(R)$ can be viewed as subrings of $S$ containing $\bar{R}=R / \tau(R)$

\section{Two-sided quotient rings in semiprime FBN rings}

The main result of this section is Theorem 8 . If $R$ is a semiprime FBN ring and $P$ is a hereditary link-closed set of prime ideals of $R$, then the left- and right-quotient rings of $R$ a.t $P$ coincide. We begin with some observations on torsion theories in a semiprime FBN ring.

Suppose $P$ is a hereditary link-closed set of prime ideals of semiprime FBN ring $R$. Let $S_{1}, S_{2}, \ldots, S_{n}$ be the minimal prime ideals of $R \quad($ Minspec $R)$. Suppose $s_{1}, s_{2}, \ldots, s_{t} \in P$ and $s_{t+1}, \ldots, s_{n} \notin P$. Let $N_{\mathrm{p}}=\bigcap_{i=1}^{t} S_{i}$. Since $\cap\left\{S_{j}: j=t+1, \ldots, n\right\}$ is P-dense and annihilates $N_{p}, N_{p} \subseteq \tau_{p}(R)$. On the other hand, $\tau_{p}(R) \subseteq P$ for all $P \in P$. Hence $\tau_{p}(R)=N_{p}$. Both $\Lambda(R)$ and $\Delta(R)$ may be viewed as subrings of $Q_{\max }\left(R / N_{p}\right)=Q_{c l}\left(R / N_{p}\right)$.

THEOREM 8. Suppose $R$ is a semiprime FBN ring. Let $P$ be a hereditary link-closed subset of spec $R$. Then the left-and rightquotient rings of $R$ coincide. 
Proof. Let $\bar{R}=R / \tau_{\mathrm{p}}(R)=R / N_{\mathrm{P}}$. Let

$Q=\operatorname{Max} P=\{Q \in P: Q$ is maximal among elements of $P\}$.

Then $Q$ is link-closed and incomparable ([7], [11]). Let $E=\Theta\left\{E_{Q}: Q \in 2\right\}$. We have $\bar{R} \subseteq E(\bar{R}) \subseteq I=\pi E$ for some product of copies of $E$. Now $\operatorname{Bic}(I) \cong \operatorname{Bic}(E)=S \cong \lim \Delta(R) / \Delta(\pi) \quad([2$, Proposition A2 $])$. By [4, Lema 3], iS/iR is 2-torsion for all $i \in I$. In particular, if $i=\bar{l}$, we get that $(\Lambda / R)_{R}$ is torsion. Since $\Lambda$ may be viewed as a subring of $Q_{\max }(\bar{R})$ and since $Q_{\max }(\bar{R}) \cong E(\bar{R}), \Lambda$ is an essential extension of $\bar{R}$. Hence $\Lambda \subseteq \Delta$. By symmetry, $\Delta \subseteq \Lambda$.

\section{Semiprime FBN rings and perfect torsion theories}

A torsion theory with right localization functor $\Delta$ is perfect (on Mod- $R$ ) if every $\Delta(R)$ module, considered as an $R$-module, is torsion-free. This is one of many characterizations of perfect torsion theories. See Stenstrom [10] and Richards [9] for more details. Call a hereditary set $P \subseteq$ Spec $R$ (or the corresponding incomparable set $Q=\max P$ ) perfect if the $P$-torsion theories on $\operatorname{Mod}-R$ and on $R$-Mod are perfect.

A torsion theory with torsion radical $\tau_{0}$ is said to decompose as a direct sum of torsion theories $\tau_{1}$ and $\tau_{2}$ if for all (right) $R$-modules $M, \quad \tau_{0}(M)=\tau_{1}(M) \oplus \tau_{2}(M)$. Müller has established the following necessary and sufficient conditions for such a decomposition. Let $D_{i}$ denote the set of $\tau_{i}$-dense prime ideals for $i=0,1,2$. Then $\tau_{0}=\tau_{1} \oplus \tau_{2}$ if $D_{0}$ is the disjoint union of $D_{1}$ and $D_{2}$ and there are no links between prime ideals in $D_{1}$ and prime ideals in $D_{2}$ [8]. Now by Goldie's theorem, Minspec $R$ is closed under links. In particular, the set of all maximal ideals which are also minimal prime ideals ("min-max" ideals) is finite and link-closed. Any link-closed set $q_{1}$ of min-max ideals induces a direct sum decomposition $R=N_{1} \oplus B$ where $N_{1}$ is the $2_{1}$-torsion submodule of $R$ [8, Theorem 3]. As noted above, $N_{1}$ is the intersection of the ideals in $Q_{1}$ and $B$ is the intersection of the 
minimal prime ideals not in $Q_{1}$. Furthermore, the $Q_{1}$-torsion theory is classical, hence perfect.

PROPOSITION 9. Let $Q$ be any link-closed set of maximal ideals in a semiprime FBN ring $R$. Let $Q_{1}=2 \cap$ Minspec $R$ and $Q_{2}=Q \backslash Q_{1}$. Let $N_{1}=\tau_{Q_{1}}(R), \quad N_{2}=\tau_{Q_{2}}(R)$ and let $B$ denote the intersection of alz minimal prime ideals not in $Q_{1}$. Let $\Delta, \Delta_{1}$, and $\Delta_{2}$ denote the zocalization functors associated with $Q_{2} Q_{1}$, and $2_{2}$ respectively. Then

$$
\Delta(R) \cong \Delta_{1}\left(R / N_{1}\right) \oplus \Delta_{2}(R / B)=\Delta_{1}(R) \oplus \Delta_{2}(R) \text {. }
$$

Proof. We have $R=N_{1} \oplus B, N_{1} \cong R / B, B \cong R / N_{1}$. It is clear that $\Delta(R) \cong \Delta\left(R / N_{1}\right) \oplus \Delta(R / B)$. We shall show that $\Delta\left(R / N_{1}\right)=\Delta_{1}(R)=\Delta_{1}\left(R / N_{1}\right)$ and $\Delta(R / B)=\Delta_{2}(R / B)=\Delta_{2}(R)$. Now ${ }^{\tau} Q_{1}(R)=N_{1}$. Hence $R / \tau_{Q_{1}}(R)=R / N_{1} \rightarrow \Delta_{1}(R)$. If $x \in \Delta_{1}(R)$, there exists a $2_{1}$-dense ideal $D$ such that $x D \subseteq R / N_{1}$. Now $R / N_{1}$ is $2_{2}-$ torsion and the $2_{2}$-torsion theory is stable. Hence there exists a $2_{2}-$ dense ideal $D^{\prime}$ such that $x D^{\prime}=0$. The prime ideals containing $D+D^{\prime}$ are both $2_{1}$ - and $2_{2}$-dense, hence 2 -dense. It follows that $x \in \Delta\left(R / N_{1}\right)=\Delta(R)$. Trivially, $\Delta\left(R / N_{1}\right) \subseteq \Delta_{1}\left(R / N_{1}\right)=\Delta_{1}(R)$ since every 2-dense ideal is $Q_{1}$-dense and $R / N_{1}$ is 2-torsion free. The proof that $\Delta(R / B)=\Delta_{2}(R / B)$ is similar after noting that $\tau_{2}(R / B)=N_{2} / B=\tau(R / B)$.

LEMMA 10. Suppose $R$ is a semiprime FBN ring with no min-max ideals. If the torsion theory associated with the hereditary set of nonmaximal prime ideals is perfect, then the torsion theory determined by any link-closed set of maximal ideals is perfect. The converse also holds.

Proof. Let $P_{0}$ denote the set of non-maximal ideals and let $Q_{1}$ be any link-closed subset of Maxspec $R$. Let $2_{2}=\operatorname{maxspec} R \backslash Q_{1}$. Denote the corresponding cohereditary sets of dense prime ideals by $D_{0}, D_{1}$ and $D_{2}$ and the corresponding torsion radicals by $\tau_{0}, \tau_{1}$ and $\tau_{2}$. Clearly, 
$D_{0}=D_{1} \cup D_{2}, D_{1} \cap D_{2}=\varnothing$ and there are no links between primes in $D_{1}$ and primes in $D_{2}$. By $\left[8\right.$, Lemma 2], $\tau_{0}=\tau_{1} \oplus \tau_{2}$. But $\tau_{0}(R)=\cap\left\{P: P\right.$ is a minimal $Q_{0}$-closed prime $\}$

$$
=\Pi\{P: P \text { is a minimal prime }\}=0 \text {. }
$$

Hence, by $[9,1.9], \tau_{1}$ and $\tau_{2}$ are perfect if and only if $\tau_{0}$ is.

REMARK. Obviously the conditions of Lemma 10 are satisfied in a semiprime ring of Krull dimension one with no min-max ideals, in particular in a bounded Noetherian prime ring of Krull dimension one.

PROPOSITION 11. Suppose $R$ is a semiprime FBN ming in which the torsion theory determined by the hereditary set of non-maximal ideals is perfect. Then every link-closed set of maximal ideals determines a perfect torsion theory.

Proof. Let $Q$ be a link-closed set of maximal ideals. Let $Q_{1}=$ Minspec $R \cap Q$ and $Q_{2}=2 \backslash Q_{1}$ as in Proposition 9 . To show that the 2-torsion theory is right perfect, it is sufficient to show that every $\Delta(R)$-module, considered as an $R$-module, is 2-torsion free (where $\Delta$ and $\Delta_{i}$ represent the localization functors corresponding to $Q$ and $q_{i}$, $i=1,2\}$. Since $\Delta(R)=\Delta_{1}(R) \oplus \Delta_{2}(R)$, any $\Delta(R)$-module $M$ is both a $\Delta_{1}(R)$ - and a $\Delta_{2}(R)$-module. If $\Delta_{1}(R) \neq 0$ then $M$ is $Q_{1}$-torsion free, hence 2-torsion free.

If $Q_{1}=\varnothing$, let $A$ denote the intersection of all min-max ideals and $B$ the intersection of the remaining minimal primes. Then $R=A \oplus B$ where $A$ is a semiprime ring containing no min-max ideals. $\Delta(R)=\Delta(R / B)$. Applying Lemma 10 to $R / B$ we get the desired result.

PROPOSITION 12. Every link-closed hereditary set of prime ideals in a semiprime FBN ring of Krull dimension one is perfect.

Proof. Let $Q=\operatorname{Max} P$ where $P$ is a hereditary link-closed subset of Spec $R$. If 2 consists entirely of maximal ideals, the conditions of Proposition 11 are satisfied. If not, let 


$$
\begin{aligned}
& Q_{1}=\{\text { min-max ideals in } 2\}, \\
& 2_{2}=\{\text { non-maximal prime ideals in } 2\}, \\
& \left.2_{3}=\text { \{non-minimal prime ideals in } 2\right\} .
\end{aligned}
$$

$Q_{1}$ and $2_{2}$ are finite link-closed (hence perfect) sets of minimal primes of $R \cdot 2_{3}$ is perfect by Proposition 1l. Let

$$
N_{i}=n\left\{S: S \text { is a minimal } Q_{i} \text {-closed prime ideal }\right\}, i=1,2,3 .
$$

There is an essential monomorphism (where $N=\tau_{2}(R)$ )

$$
R / N \stackrel{h}{\longrightarrow} R / N_{1} \oplus R / N_{2} \oplus R / N_{3}
$$

Let

$$
0 \rightarrow R / N \rightarrow \bigoplus_{i=1}^{3} R / N_{1} \rightarrow X \rightarrow 0
$$

be exact. Applying the exact functor $\Delta=\Delta_{Q_{i}}$ for $i=1,2,3$, we have exact sequences

$$
0 \rightarrow \Delta_{i}(R / N) \rightarrow \bigoplus_{j=1}^{3} \Delta_{i}\left(R / N_{j}\right)+\Delta_{i}(X) \rightarrow 0
$$

But $\Delta_{i}\left(R / N_{j}\right)=0$ for $i \neq j$. Indeed, $R / N_{j}$ is $Q_{i}$-torsion for $i \neq j$, and the $2_{i}$-torsion theory is stable. Also $\Delta_{i}(R / N)=\Delta_{i}\left(R / N_{i}\right)$. Hence $\Delta_{i}(X)=0$, for $i=1,2,3$, from which we get that $X$ is 2-torsion. In other words, $R / N$ is a 2-dense submodule of $\oplus_{j=1}^{3} R / N_{j}$. It follows that $\Delta(R / N) \cong \Delta\left(R / N_{1}\right) \oplus \Delta\left(R / N_{2}\right) \oplus \Delta\left(R / N_{3}\right)$. By the proof of Proposition 9, $\Delta\left(R / N_{i}\right)=\Delta_{i}\left(R / N_{i}\right)$ for $i=1,2,3$. Consequently, the perfectness of $Q_{1}, 2_{2}$, and $2_{3}$, implies perfectness of 2 .

REMARK 1. Proposition 12 shows that at least in the case of a semiprime FBN ring, the assumption of zero socle in [7, Proposition 15] can be dropped. We do not know whether this is true for non-semiprime $R$. 
REMARK 2. It is an open question when the hypotheses of Lemma 10 and Proposition 11 are satisfied. If $R=k[x, y]$ for some field $k$, then $R$ is a prime FBN ring of Krull dimension 2 in which the hypotheses of Lemma 10 are not satisfied (see [7]).

\section{References}

[1] J.A. Beachy, "Stable torsion radicals over FBN rings", J. Pure Appl. Algebra 24 (1982), 227-233.

[2] C. Faith, Injective modules and injective quotient rings (Lecture Notes in Pure and Applied Mathematics, 72. Marcel Dekker, New York, 2982).

[3] A.V. Jategaonkar, "Jacobson's conjecture and modules over fully bounded Noetherian prime rings", J. Algebra 30 (1974), 103-121.

[4] J. Lambek, "Bicommutators of nice injectives", J. Algebra 21 (1972), $60-73$.

[5] J. Lambek and G. Michler, "Localization of right Noetherian rings at semiprime ideals", Canad. J. Math. 26 (1974), 1069-1085.

[6] B.J. Müller, "Localization in fully bounded Noetherian rings", Pacific J. Math. 67 (1976), 233-245.

[7] B.J. Müller, "Two-sided localization in Noetherian PI rings", $J$. AZgebra 63 (1980), 359-373.

[8] B.J. Müller, "Links between maximal ideals in bounded Noetherian prime rings of Krull dimension ane", preprint.

[9] R. Richards, "Noetherian prime rings of Krull dimension 1 " ( $\mathrm{PhD}$ Thesis, McMaster University, Hamilton, Ontario, 1977).

[10] B. Stenstrom, Rings and modules of quotients (Lecture Notes in Mathematics, 237. Springer-Verlag, Berlin, Heidelberg, New York, 1971).

[11] M. Upham, "Right localizable multiplicative sets and prime ideals of FBN rings", Comm. Algebra 9 (1981), 1093-1103.

Department of Mathematics, Southern III inois University, Carbondale, IIlinois 62901, USA. 